\title{
Posibilidades del uso de Trichoderma harzianum en el biocontrol del marchitamiento y cancro bacteriano del tomate
}

\section{Possibilities of the use of Trichoderma harzianum in the biocontrol of wilt and bacterial canker of tomato}

\section{Jorgelina Rolleri *}

Centro de Investigaciones de Fitopatología. Facultad de Ciencias Agrarias y Forestales, Universidad Nacional de La Plata; Ministerio de Desarrollo Agraria (PBA), Argentina

\section{Marina Stocco}

Centro de Investigaciones de Fitopatología. Facultad de Ciencias Agrarias y Forestales, Universidad Nacional de La Plata, Argentina

\section{Paulina Moya}

Instituto de Ciencias Polares, Ambiente y Recursos Naturales de la Universidad Nacional de Tierra del Fuego, Antártida e Islas del Atlántico Sur

\section{Cecilia Mónaco}

Centro de Investigaciones de Fitopatología. Facultad de Ciencias Agrarias y Forestales, Universidad Nacional de La Plata, Argentina

\section{Revista de la Facultad de Agronomía}

Universidad Nacional de La Plata, Argentina

ISSN: 1669-9513

Periodicidad: Semestral

Vol. 120, núm. 2, 2021

redaccion.revista@agro.unlp.edu.ar

Recepción: 03/09/20

Aprobación: 09/11/20

URL: http://portal.amelica.org/ameli/jatsRepo/23/232371007/index.html

DOI: https://doi.org/10.24215/16699513e080

* Autor de correspondencia: jorgelinarolleri@gmail.com 


\title{
Resumen
}

El presente trabajo consistió en evaluar la posibilidad del uso de dos cepas de Trichoderma harzianum (Th118 y Th5cc) sobre la manifestación del marchitamiento y cancro bacteriano del tomate en plantas de tomate platense cultivadas en invernáculo. Ambas cepas se aplicaron al sustrato de la plantinera en forma sólida (S) y líquida (L) en el momento de la siembra. Clavibacter michiganensis subsp. michiganensis se inoculó en el momento del desbrote mediante una pequeña incisión en el tallo principal entre la quinta y sexta hoja. El ensayo se repitió durante dos años consecutivos. Para cada tratamiento se evaluaron los componentes del rendimiento (peso y número de frutos) y número de hojas afectadas. Si bien no se encontraron diferencias significativas entre tratamientos durante los dos años, el tratamiento Th118 L es el que presentó menor cantidad de hojas afectadas y valores promedio más altos de peso de frutos. De acuerdo con los resultados obtenidos, se puede afirmar que la cepa de Trichoderma harzianum Th 118 aplicada como riego a los plantines en el momento de la siembra, podría ser incorporada como una buena alternativa dentro de un plan de manejo integrado de enfermedades en el cultivo de tomate platense.

Palabras clave: biocontrol, Clavibacter michiganensis subsp michiganensis, tomate platense, antagonistas, cultivo en invernáculo

\begin{abstract}
The objective of this work was to evaluate the possibility of using two strains of Trichoderma harzianum (Th118 and Th5cc) against bacterial wilt and canker symptoms in tomato plants grown under greenhouse conditions. Both strains were applied on the substrate of the tomato seedling in solid (S) and liquid (L) form at sowing. Clavibacter michiganensis subsp. michiganensis was inoculated at the time of budding through a small incision in the main stem between the fifth and sixth leaves. The trail was repeated for two consecutive years. For each treatment, the yield components (number and weight of fruits) and number of affected leaves were evaluated. Although no significant differences were found between treatments during the two years, the Th118 L treatment was the only that presented the least amount of affected leaves and higher average values of fruit weight. According to results obtained, it can be stated that the Trichoderma harzianum Th118 strain applied as irrigation to the seedlings at the time of sowing could be incorporated as a good alternative within an integrated disease management plan in the tomato crops.
\end{abstract}

Keywords: biocontrol, Clavibacter michiganensis subesp. michiganensis, tomato var. "platense", antagonists, greenhouse crops 


\section{INTRODUCCIÓN}

El tomate (Solanum lycopersicum L. = Lycopersicon esculentum Mill.) es una planta dicotiledónea perteneciente a la familia de las solanáceas (Peralta et al., 2005). Es una de las hortalizas más consumidas y distribuidas en todas las regiones del mundo. Es la hortaliza más importante por su consumo, superficie cultivada y por la tecnología desarrollada en torno a ella. La producción mundial de tomate fresco es de 177.042.056 t (FAOSTAT, 2016); se encuentra dentro de los diez alimentos de mayor producción a nivel mundial. En Argentina se cultivan 17.800 ha de tomate (INTA \& CMCBA, 2009), de las cuales 10.500 ha se destinan a mercado fresco y 6.500 ha para industria. Es la hortaliza que ocupa la mayor superficie cultivada bajo cubierta del país, representando el $40 \%$ del total (Corvo Dolcet, 2005), concentrada principalmente en las provincias de Buenos Aires y Corrientes. Se producen en el país aproximadamente 1.000 .000 de $t$ de las cuales 650.000 son comercializadas para tomate en fresco (Del Pino, 2020). El Cinturón Hortícola Platense (CHP), que comprende las zonas productivas de los partidos de La Plata, Berazategui y Florencio Varela, no sólo es la región más importante de la provincia de Buenos Aires, sino que además es una de las zonas productivas más capitalizadas de Argentina. En los últimos 20 años la continua incorporación de invernaderos ha permitido su crecimiento en producción a nivel regional, provincial y nacional (García, 2011). Tiene más de 4.000 ha bajo cubierta y produce el $67 \%$ del tomate bajo invernadero de toda la provincia (CHFBA, 2005; MDA, 2018).

El tomate platense es una variedad botánica caracterizada por su sabor intenso, su forma irregular, achatada, acostillada o fuertemente lobulada (Garat, 2002). Es un producto típico de esta región (CHP) construido desde hace más de 70 años a través de una relación muy fuerte entre actores y territorio, dando por resultado un producto reconocido por su sabor, aroma y rusticidad. Como tal ha dominado por décadas la producción de tomate fresco en la región rioplatense, pero desde la década del 80 , ha sido paulatinamente desplazado por materiales genéticos modernos, hasta tener una presencia menor tanto en las quintas como en el mercado. No obstante, en los últimos años se han desarrollado experiencias en el ámbito internacional y nacional, vinculadas a la revalorización y conservación de los materiales genéticos locales. Esto permitió trazar la existencia de un buen número de variedades de tomate platense de la región (Garat, 2002), las cuales, como llevan algunos años de producción continua en la zona, están adaptadas a las condiciones de suelo y clima. Esta característica y la experiencia reciente permiten inferir que la incorporación de variedades de tomate platense a las rotaciones sería beneficioso para disminuir la dependencia de insumos externos, respecto a las variedades comerciales, especialmente en el uso de agroquímicos (Otero et al., 2014).

El marchitamiento y cancro bacteriano del tomate es producido por la bacteria Clavibacter michiganensis subsp. michiganensis (Cmm) (Davis et al.,1984; Smith, 1910). Se encuentra presente en prácticamente todas las zonas productoras de tomate del mundo (EPPO/CABI, 1998; De León et al., 2011; $\mathrm{CABI}, 2015)$. Esta enfermedad ha causado grandes pérdidas en los cultivos de tomate tanto a campo como en invernadero. El síntoma más relevante es el marchitamiento y muerte de las plantas, lo que ocasiona grandes pérdidas económicas (Tancos et al., 2013; CABI, 2015; Chalupowicz et al., 2016). Cuando se presenta puede enfermar a todas las plantas en parcelas o invernaderos en poco tiempo (Kawaguchi et al., 2010; EPPO, 2013). Es de muy difícil control y las prácticas de manejo empleadas se han enfocado principalmente a medidas preventivas y al uso de productos de síntesis químicas (cúpricos y antibióticos), sin embargo, estos no han sido las prácticas más eficientes. Al contrario, no sólo aumentan los costos de producción, sino que causan daños al hombre, animales y al ambiente.

Impulsada por una creciente conciencia con su entorno, día a día la sociedad se vuelve más exigente con lo que consume, por lo que demanda una oferta mayor de alimentos con atributos de calidad específicos, más sanos y obtenidos de forma ambientalmente sustentable. Actualmente, el control de cualquier enfermedad implica buscar alternativas que permitan disminuir el uso de agroquímicos y de esta manera contribuir al cuidado del hombre y el ambiente.

Existen otras prácticas para el manejo de las enfermedades de los cultivos que actúan de manera más amigable con el hombre y su ambiente, como lo es el control biológico. Éste se basa en la incorporación de microorganismos benéficos que pueden establecer interacciones antagónicas con los patógenos, o bien estimular los mecanismos de defensa del hospedante (Montesinos \& Bardají, 2008). En este sentido, se han reportado resultados positivos del uso de Trichoderma spp. sobre el crecimiento y productividad en diversas especies hortícolas (Grondona et al., 1997; Brunner et al., 2005; Vinale et al., 2008; Kariuki et al., 2020). Trichoderma harzianum Rifai es un hongo de vida libre que vive en el suelo y coloniza las raíces de muchas plantas como un simbionte oportunista no virulento (Harman et al., 2004). Se ha utilizado en 
aplicaciones biotecnológicas como biofungicida y como promotor de crecimiento ya que proporciona beneficios importantes para la agricultura en condiciones de campo (Yedidia et al., 2001; Bal \& Altintas, 2006; Lorito et al., 2010). Existen reportes sobre la incorporación de este hongo en tomate, durante la etapa de almácigo, donde promovió el crecimiento, tanto de la parte aérea como radical, redundando en la obtención de plantines de mejor calidad al trasplante (Moo-Koh et al., 2017). En este sentido, González et al. (2013) y Jiménez et al. (2011) reportaron incrementos en el rendimiento al aplicarlo luego de la siembra. Mientras que Galeano et al. (2002) observaron que al incorporar T. harzianum previo al trasplante en plantas de tomate, pimiento y pepino, presentaban mayor altura respecto a las plantas no inoculadas.

Guerrero et al. (2017a; 2017b) y Utkhede \& Koch (2004) señalaron una reducción del cancro bacteriano del tomate en ensayos en invernáculo mediante la incorporación de $T$. harzianum tanto al sustrato como a la parte aérea de plantas de tomate. Adicionalmente, dada su versatilidad al momento de actuar frente a los patógenos Trichoderma sp. ofrece un mayor rango de acción que no ha sido superado fácilmente por otros agentes de control biológico (Harman et al., 2004; Samuels, 2006).

El objetivo del presente trabajo fue evaluar la posibilidad del uso de Trichoderma harzianum, bajo dos modalidades de aplicación, sólida y líquida, sobre la manifestación del marchitamiento y cancro bacteriano y el rendimiento en plantas de tomate platense cultivadas bajo cubierta.

\section{MATERIALES Y MÉTODOS}

\section{APLICACIÓN DE LOS ANTAGONISTAS}

Se utilizaron dos cepas de T. harzianum (Th118 y Th5cc) como antagonistas. La cepa Th118 fue aislada del filoplano de hojas de tomate y probada previamente en ensayos de invernáculo donde redujo la incidencia de la enfermedad ocasionada por Botrytis cinerea (Dal Bello et al., 2011). Por otra parte, la cepa Th5cc fue aislada del filoplano de trigo y usada previamente como antagonista de Zymoseptoria tritici (Cordo et al., 2007). Además, en trabajos previos se determinó que la cepa Th5cc aplicada como formulación líquida y como recubrimiento en semillas, resultó ser la más efectiva en mantener una alta población de T. harzianum en el suelo con un potencial efecto biocontrolador (Stocco et al., 2019). Ambas cepas se identificaron molecularmente siguiendo la técnica descripta por Stocco et al. (2016) y fueron depositadas en la base de datos del European Molecular Biology Laboratory (EMBL) bajo los números de acceso LN869400 ( $T$. harzianum Th118) y LN869401 ( $T$. harzianum Th5cc). A su vez, estas cepas se encuentran depositadas en el cepario perteneciente al Centro de Investigaciones de Fitopatología (CIDEFI).

La incorporación de este hongo se realizó al sustrato de los plantines de dos maneras diferentes, como preparación sólida y como preparación líquida. Para incorporar cada uno de los antagonistas de forma sólida, $1 \mathrm{ml}$ de una suspensión de esporas de cada cepa de $T$. harzianum $\left(10^{8}\right.$ esporas $\left./ \mathrm{ml}\right)$ se sembró en $100 \mathrm{~g}$ de una mezcla de arena, salvado de trigo y agua en partes iguales, previamente esterilizada durante dos días consecutivos. Se incubó a temperatura ambiente $\left(25+/-2^{\circ} \mathrm{C}\right)$ durante 10 días. Posteriormente, la mezcla colonizada por el antagonista se secó en bolsas de papel dobles en estufa a $40{ }^{\circ} \mathrm{C}$ durante $24 \mathrm{~h}$. El inóculo fúngico se desmenuzó manualmente y se incorporó al sustrato de los plantines en una proporción de 1:100 (por cada $2 \mathrm{~kg}$ de sustrato se utilizaron $20 \mathrm{~g}$ de inóculo). Por último, se sembraron las semillas de tomate en speedlings, una por cada celda.

El tratamiento testigo consistió en sembrar semillas de tomate sobre sustrato sin inóculo de T. harzianum. Para la incorporación del antagonista en forma líquida, se preparó una suspensión de esporas de cada cepa de $T$. harzianum desarrolladas en medio PDA (papa dextrosa agar) durante 8 días de crecimiento. La suspensión se realizó agregando agua estéril sobre la caja de Petri colonizada y raspando con un ansa estéril. Luego se ajustó a 1 x 108 esporas/ml y se agregó Tween $20(0,01 \%)$. El inóculo fúngico se aplicó por única vez en el momento de la siembra en forma de riego utilizando $10 \mathrm{ml}$ de suspensión por cada cavidad de la bandeja de siembra. Por último, se sembró una semilla de tomate en cada celda. En el momento de la siembra este tratamiento se regó con $10 \mathrm{ml}$ de agua destilada estéril.

\section{PREPARACIÓN DEL INÓCULO DE CLAVIBACTER MICHIGANIENSIS SUBSP. MICHIGANIENSIS}

Para este trabajo se utilizó la cepa de la bacteria patógena Cmm (LPAb158), que se encuentra depositada en el cepario del Centro de Investigaciones de Fitopatología (CIDEFI). La misma fue identificada por técnicas microbiológicas y moleculares usando cebadores específicos que amplifican la región 
intergénica 16S-23S del ARNr (Patrik \& Rainey, 1999; Schaad et al., 2001; EPPO, 2013), descripto por Rolleri (2015).

Para la inoculación se partió de una suspensión bacteriana conservada en caldo nutritivo y glicerol (20\%) y almacenada a $-20^{\circ} \mathrm{C}$. La cepa bacteriana, se cultivó en cajas de Petri, en medio Agar Nutritivo (AN), se sembró en estrías por agotamiento y se incubó a $27^{\circ} \mathrm{C}\left(+/-2^{\circ} \mathrm{C}\right)$ entre 48 a $72 \mathrm{~h}$. Luego, se realizó una suspensión de las colonias en agua destilada estéril, ajustándose a una lectura en el espectrofotómetro de $\mathrm{OD}_{600}=0,3\left(\sim 5,5 \times 10^{8} \mathrm{UFC} / \mathrm{ml}\right)$ diluyéndose $1: 10$ para alcanzar la concentración final de uso de 107 ufc/ml.

\section{ENSAYOS EN INVERNÁCULO}

Los ensayos se llevaron a cabo en un invernáculo (de $6 \mathrm{~m} \times 20 \mathrm{~m}$, con mampostería de madera y polietileno de $180 \mu$ de espesor) situado en la Chacra Experimental Gorina, del Ministerio de Desarrollo Agrario de la provincia de Buenos Aires ( $34^{\circ} 54^{\prime} 56,4^{\prime \prime} \mathrm{S} 58^{\circ} 02^{\prime} 21,5^{\prime \prime} \mathrm{W}$ ), perteneciente al Cinturón Hortícola Platense. Los plantines se sembraron en bandejas de siembra con los tratamientos respectivos descriptos anteriormente y se trasplantaron al invernáculo cuando tenían entre 10 y $15 \mathrm{~cm}$ de altura y al menos 4-6 hojas verdaderas desplegadas.

Se realizó una sola inoculación con la bacteria patógena $\mathrm{Cmm}$ en el momento del primer desbrote cuando las plantas tenían entre 12-14 hojas. La misma consistió en sumergir la cuchilla de desbrote en la suspensión bacteriana con una concentración de $10^{8} \mathrm{UFC} / \mathrm{ml}$ realizando un pequeño tajo en el tallo principal de la planta entre la quinta y sexta hoja.

Se realizó un ensayo durante la campaña 2015-2016 (ensayo 1) el cual se repitió en 2016-2017 (ensayo 2) en el mismo invernáculo.

\section{DISEÑO EXPERIMENTAL Y ANÁLISIS ESTADÍSTICO}

El diseño experimental consistió en 3 bloques con 5 tratamientos al azar y 10 repeticiones de cada uno. Los tratamientos fueron:

-Tratamiento 1: Aplicación de la cepa Th118 en forma líquida (aplicada como riego) a la siembra y posterior inoculación con la suspensión bacteriana de $\mathrm{Cmm}$ en las plantas de tomate en el invernáculo (Th118 L).

-Tratamiento 2: Incorporación de la cepa Th118 en forma sólida (aplicada al sustrato) a la siembra y posterior inoculación con la suspensión bacteriana de $\mathrm{Cmm}$ en las plantas de tomate en el invernáculo (Th118 S).

-Tratamiento 3: Aplicación de la cepa Th5 cc en forma líquida a la siembra y posterior inoculación con una suspensión bacteriana de $\mathrm{Cmm}$ en las plantas de tomate en el invernáculo (Th5 cc L).

-Tratamiento 4: Incorporación de la cepa Th5 cc en forma sólida a la siembra y posterior inoculación con una suspensión bacteriana de $\mathrm{Cmm}$ en plantas de tomate en el invernáculo (Th5 cc S).

-Tratamiento 5: (Testigo) Tomate más inoculación de suspensión bacteriana $\mathrm{Cmm}$ sin aplicación de antagonista.

Para cada tratamiento se evaluaron parámetros relacionados con la sanidad y el rendimiento de los frutos. Con respecto a la sanidad de las plantas se evaluó a los 30 días posteriores a la inoculación, el número de hojas con síntomas sobre el total de hojas por planta. Respecto al rendimiento de frutos se evaluó peso y número de frutos por planta al momento de la maduración de la primera corona.

El número de hojas se analizó mediante análisis de la varianza (ANOVA), el peso y número de frutos se analizó mediante prueba no paramétrico de Friedman, debido a que los datos no cumplían con los supuestos de normalidad y varianzas homogéneas. Para los análisis estadísticos se utilizó el programa Infostat® (Di Rienzo et al., 2015).

\section{RESULTADOS}

\section{ENSAYOS EN INVERNÁCULO}

No se observaron diferencias significativas en el número de hojas con síntoma de marchitamiento y cancro bacteriano entre los tratamientos tanto para el ensayo 1 ( $F: 0,55 ; p$-valor $=0,7)$ como para el ensayo 2 (F: 1,74; $p$-valor =0,15). Para el primer año en los tratamientos 1, 3 y 4 (Th118 L, Th5 cc L y Th5cc S) la enfermedad respecto al testigo fue menor. En el segundo año todos los tratamientos con $T$. harzianum presentaron menos hojas afectadas que el testigo, siendo el tratamiento Th118 L el que presentó menor cantidad de hojas afectadas por la enfermedad (Figura 1). 
Con respecto al peso de los frutos, no hubo diferencias significativas entre el testigo y los tratamientos con T. harzianum en los dos ensayos. En el ensayo 1, si bien el análisis estadístico no mostró diferencias significativas entre los tratamientos (T: 2,20; $p$-valor $=0,07)$ el tratamiento Th5cc $L$ y el testigo fueron los que presentaron menores valores de peso (promedio 315 y $480 \mathrm{~g}$ respectivamente). El tratamiento Th 118 $\mathrm{L}$ fue el que se destacó presentando mayores valores de peso de frutos (promedio $580 \mathrm{~g}$ ). Para el ensayo 2 tampoco se registraron diferencias significativas entre todos los tratamientos (T: 1,49; $p$-valor=0,21) aunque el testigo registró los menores valores de peso de frutos (promedio $470 \mathrm{~g}$ ). El tratamiento Th $5 \mathrm{cc} L$ y Th $118 \mathrm{~L}$ revelaron los valores más altos de peso de frutos cosechados (promedio 1000 y $900 \mathrm{~g}$ respectivamente) (Figura 2).

En cuanto al número de frutos hubo diferencias para esta variable en los dos ensayos. Para el ensayo 1 el análisis estadístico muestra diferencias significativas entre los tratamientos (T: 3,42; $p$-valor $=0,01)$. El testigo y el tratamiento Th $5 \mathrm{cc} L$ registraron la menor cantidad de frutos. Los tratamientos Th118 $\mathrm{L}$ y Th118 $S$ fueron los que presentaron mayor cantidad de frutos (Figura 3).

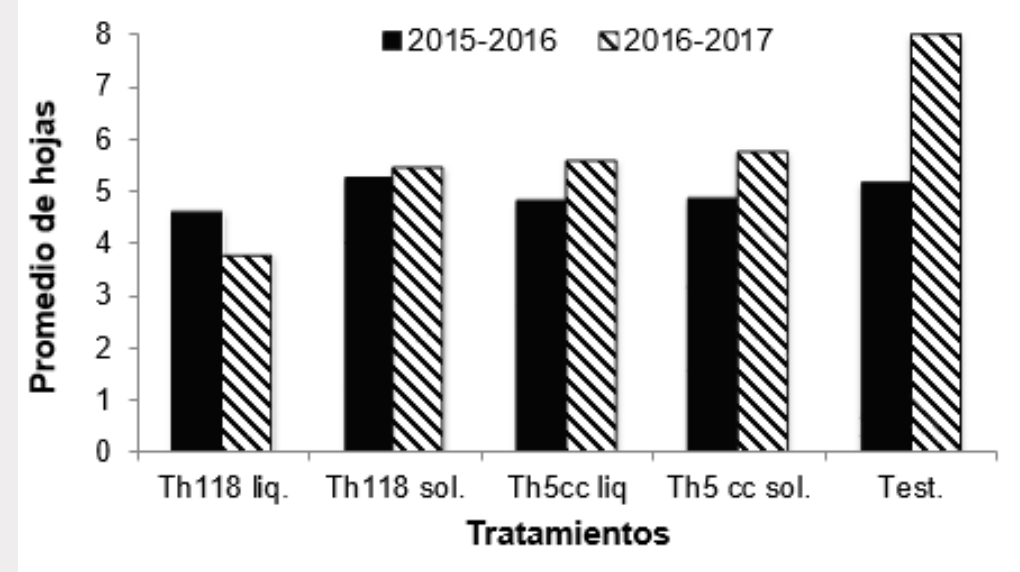

Figura 1

Promedio del número de hojas afectadas por cancro bacteriano en cada tratamiento con T. harzianum y testigo para cada ensayo. No se indican letras ya que no existen diferencias significativas entre tratamientos para cada ensayo según el análisis de ANOVA.

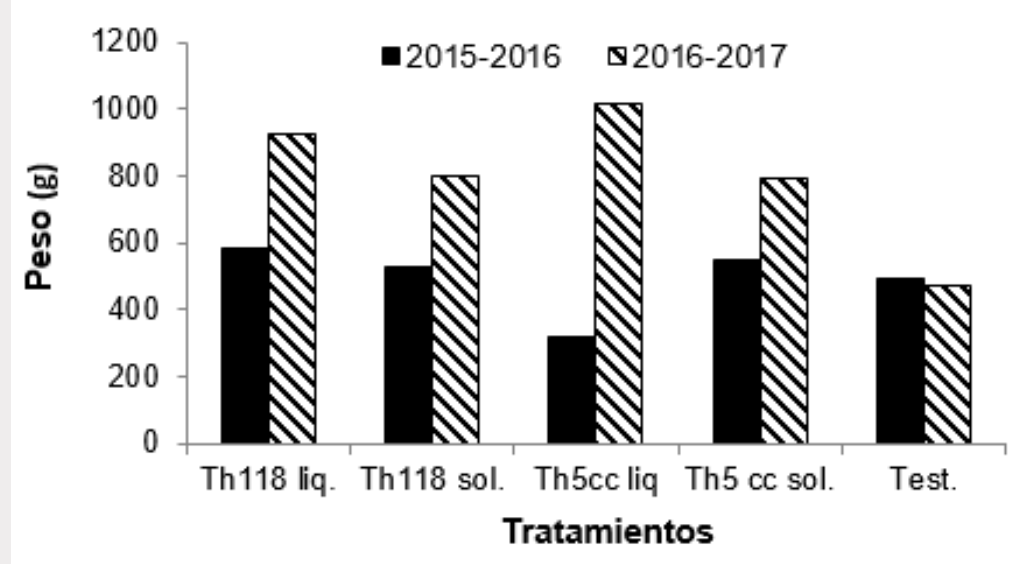

Figura 2

Promedio del peso de frutos en cada tratamiento con T. harzianum y testigo para cada ensayo. No se indican letras ya que no existen diferencias significativas entre tratamientos para cada ensayo de acuerdo a la prueba de Friedman. 


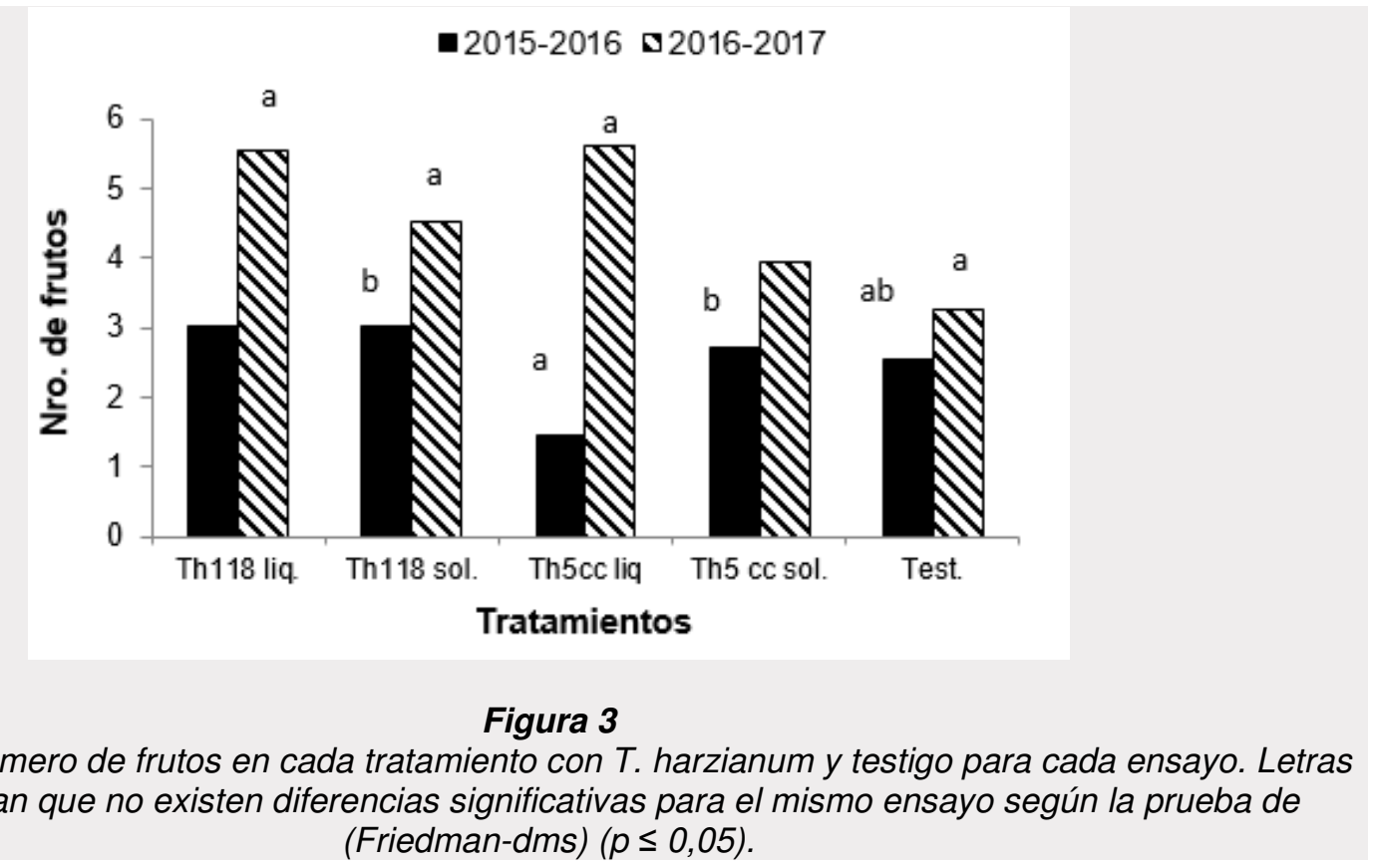

En el ensayo 2, los tratamientos no presentaron diferencias significativas para la variable número de frutos (T: 1,74; $p$-valor $=0,15)$ aunque el testigo fue el que registró menor cantidad de frutos cosechados. Los tratamientos Th118 L y Th5cc L fueron los que produjeron mayor cantidad de frutos (Figura 3).

Según lo observado en los resultados, hubo una tendencia favorable del tratamiento Th118 $\mathrm{L}$ a mejorar tanto los parámetros de sanidad de las plantas de tomate como de rendimiento. Este tratamiento fue el que presentó menor cantidad de hojas afectadas, así como también mayores valores de peso y número de frutos cosechados.

\section{DISCUSIÓN}

En los últimos años, en el mundo ha aumentado el interés en el control biológico de patógenos vegetales, especialmente de bacterias fitopatógenas (Volksch \& May 2001; Wilson et al., 2002; Nguyen \& Ranamukhaarachchi, 2010; Valdez Morales, 2016; Zahir et al., 2018; Abo-Elyousr et al., 2019). Dentro de este contexto, y de acuerdo con los resultados de estas investigaciones, el uso de T. harzianum es una buena alternativa para controlar el marchitamiento y cancro bacteriano del tomate, y así reducir las consecuencias ambientales de las aplicaciones de productos tóxicos.

En este trabajo se observó que el promedio de hojas afectadas por la bacteria patógena disminuyó al ser tratadas con el antagonista, esto coincide con los resultados de Anwar \& lqbal (2017). Estos autores estudiaron la actividad antimicrobiana de $T$. harzianum contra las bacterias Xanthomonas campestris y Clavibacter michiganensis y encontraron que el hongo produce metabolitos antibacterianos que en condiciones de laboratorio disminuyen el crecimiento de las bacterias fitopatógenas y que esto depende de las condiciones del medio (temperatura, $\mathrm{pH}$, medio de cultivo y humedad). Además, Noshad et al. (2019) estudiaron la actividad antibacteriana del hongo antagonista sobre Clavibacter michiganensis, Agrobacterium tumefaciens y Xanthomonas campestris y observaron que utilizando nanoparticulas de plata a partir de una suspensión de esporas de $T$. harzianum se inhibía el crecimiento de las bacterias fitopatógenas.

Además de sus capacidades antimicrobianas como agente de biocontrol, este hongo tiene otros efectos directos en las plantas de tomate. Estos incluyen el aumento en el rendimiento y el efecto duradero de esta protección ya que el antagonista se incorporó en los plantines y su efecto duró hasta la cosecha. 
En pruebas realizadas en plantines de tomate Guerrero et al. (2017b) señalan una reducción de hasta un $50 \%$ en la severidad del marchitamiento y cancro bacteriano del tomate en plantas infestadas con $T$. harzianum, en invernadero. Estos autores observaron también un aumento en el peso seco y fresco (entre 11 y $13 \mathrm{~g}$ ) de las raíces de las plantas tratadas con este antagonista demostrando así, la capacidad de $T$. harzianum para mejorar la sanidad de las plantas.

La incorporación del agente de biocontrol al sustrato en el momento de la siembra, tiene como objetivo permitir que los conidios de T. harzianum colonicen el plantín, y al momento del trasplante, ya se haya puesto en contacto con la planta. Este hecho queda demostrado por los resultados promisorios que se observaron al inocular las plantas con Th 118 L. En este sentido, y como observaron Cordo et al., (2007) y Stocco et al., (2015), las plantas tratadas previamente con $T$. harzianum además de provocar menos severidad de la enfermedad demostraron un mayor desarrollo radicular. Esta afirmación y en concordancia con Bailey et al., (2011), podría deberse a que un mayor desarrollo de las raíces tiene el beneficio adicional de elevar la capacidad de soportar condiciones de estrés en las plantas colonizadas. Al respecto, Brotman et al., (2013) y Hermosa et al., (2012) coinciden en que las cepas de Trichoderma sp. pueden interactuar directamente con las raíces colonizando el apoplasto, incrementando el potencial de crecimiento, la resistencia a enfermedades y la tolerancia a estreses abióticos. Asimismo, Recinos Cabrera, (2015) observó que los mecanismos de acción de $T$. harzianum como promotor del crecimiento vegetal se manifiestan desde las primeras fases de la plántula, favoreciendo una mayor capacidad exploratoria del sistema radicular que le confiere ventajas al plantín en el momento del trasplante.

Teniendo en cuenta que el antagonista se incorporó al sustrato y $\mathrm{Cmm}$ se inoculó en la parte aérea de la planta, la disminución de los síntomas de la enfermedad también podría deberse a que los aislamientos de $T$. harzianum inducen diferentes tipos de señales que dan como resultado la activación de genes, la producción de enzimas involucradas en la supresión del patógeno y el incremento de barreras bioquímicas y estructurales en la planta (Hoitink et al., 2006; Hermosa et al., 2012; Nawrocka \& Malolepsza, 2013). Este efecto sumado al mejoramiento del vigor de las plantas tiene un papel importante al momento de desarrollarse la infección y la posterior manifestación de los síntomas.

Con respecto al comportamiento diferencial de la cepa $T$. harzianum (Th $5 \mathrm{cc}$ ) en los distintos años y, habida cuenta que el tomate platense no es un híbrido, y por lo tanto cada planta tiene una respuesta distinta ante una misma cepa, podemos sugerir que esta cepa tuvo un efecto diferente teniendo en cuenta lo reportado por Lo \& Lin (2002) y Tucci et al., (2011). Estos autores observaron que, inclusive cepas de la misma especie muestran diferentes grados de acción sobre un cultivo y que también pueden variar de un cultivar a otro. Los autores señalan que esto podría estar influenciado por factores aún desconocidos. En concordancia con lo antes dicho, y como lo expresa Baker (1991) estos factores podrían estar relacionados con la habilidad saprofítica competitiva o la afinidad para sobrevivir en la rizósfera lo que podría haber sucedido con la cepa Th5cc en los distintos años de ensayo. De acuerdo a los resultados obtenidos en este trabajo, y al amplio potencial de acción que tiene el hongo $T$. harzianum como antagonista de fitopatógenos es necesario profundizar la investigación, tanto para la cepa Th5cc, por su comportamiento diferencial, como para la Th118. Esta última, al ser aplicada como riego a los plantines podría ser incorporada como una buena alternativa dentro de un plan de manejo integrado de enfermedades en el cultivo de tomate platense.

\section{BIBLIOGRAFÍA}

Abo-Elyousr, K.A.M., H.M.M. Khalil Bagy, M. Hashem, S.A.M. Alamri \& Y.S. Mostafa. 2019. Biological control of the tomato wilt caused by Clavibacter michiganensis subsp. michiganensis using formulated plant growth-promoting bacteria. Egyptian Journal of Biological Pest Control 29: 54.7.

Anwar, J. \& Z. Iqbal. 2017. Effect of growth conditions on antibacterial activity of Trichoderma harzianum against selected pathogenic bacteria. Sarhad Journal of Agriculture 33: 501-510.

Bailey, B., H. Bae, R. Melnick \& J. Crozier. 2011. The endophytic Trichoderma hamatum isolate DIS 219 $b$ enhances seedling growth and delays the onset of drought stress in Theobroma cacao. En: Endophytes of Forest Trees: Biology and Applications. M. Pirttila, C. Frank, M. Pirttila, \& C. Frank, Ed, Netherlands: Springer. pp: 157-172. 
Baker, R. 1991. Induction of rhizosphere competence in biocontrol fungus Trichoderma spp. In The Rhizosphere and Plant Growth. Keiter D.L. \& P. Cregan, eds, Kluwer Academic Publishers, Boston, pp: 221-228.

Bal, U. \& S. Altintas. 2006. A positive side effect from Trichoderma harzianum, the biological control agent: Increased yield in vegetable crops. Journal of Environmental Protection and Ecology 7: 383-387.

Brotman, Y., U. Landau, A. Cuadros-Inostroza, T. Tohge, T. Takayuki, A. Fernie \& L. Willmitzer. 2013. Trichoderma-plant root colonization: escaping early plant defense responses and activation of the antioxidant machinery for saline stress tolerance. PLoS Pathogens 9: 1-15.

Brunner, K., S. Zeilinger, R. Ciliento, S.L. Woo, M. Lorito, C.P. Kubicek \& R. Mach. 2005. Improvement of the fungal biocontrol agent Trichoderma artoviride to enhance both antagonism and induction of plant systemic disease resistance. Applied Environmental Microbiology 71: 3959-3965.

CABI. 2015. Centre for Agricultural Bioscence International. Datasheet: Clavibacter michiganensis subsp. michiganensis (bacterial canker of tomato). Disponible en https://www.cabi.org/isc/datasheet/15338. Ultimo acceso: octubre de 2020.

Chalupowicz, L., I. Barash, M. Reuven, O. Dror, G. Sharabani, K.H. Gartemann \& S. ManulisSasson. 2016. Differential contribution of Clavibacter michiganensis subsp. michiganensis virulence factors to systemic and local infection in tomato. Molecular Plant Pathology 18: 336-346.

CHFBA. 2005. Censo Horti- Florícola Provincial de Buenos Aires. Ministerio de Asuntos Agrarios de la Provincia de Buenos Aires, Consejo Federal de Inversiones y Secretaria de Agricultura, Ganadería, Pesca y Alimentación de la Nación. Disponible http://www.estadistica.ec.gba.gov.ar/dpe/Estadistica/chfba/chfba2005.pdf. Ultimo acceso: septiembre de 2021.

Cordo, C., C. Mónaco, C. Segarra, A. Perelló, D. Bayo, A. Mansilla, N. Kripelz \& R. Conde. 2007. Trichoderma spp. as elicitors in the defense responses of wheat plants against Septoria tritici. Biocontrol Science and Technology 17: 687-698.

Corvo Dolcet, S. 2005. Zonas de producción del cultivo de tomate en la Argentina. Secretaría de Agricultura, Ganadería, Pesca y Alimentos. pp: 15. Disponible en https://www.seedquest.com/News/releases/2005/pdf/13528.pdf. Ultimo acceso: septiembre de 2021.

Dal Bello, G., M.C. Rollán, G. Lampugnani, C. Abramoff, L. Ronco, S. Larran, M. Stocco \& C. Mónaco. 2011. Biological control of leaf grey mould of greenhouse tomatoes caused by Botrytis cinerea. International Journal of Pest Management 57: 177-182.

Davis, M. J., A.G.Jr. Gillaspie, A.K. Vidaver \& R. Harris. 1984. Clavibacter: a new genus containing some phytopathogenic Coryneform bacteria, including Clavibacter xyli subsp. xyli sp. nov., subsp. nov., and Cavibacter xyli subsp. cynodonis subsp. nov., pathogens that cause Ratoon stunting disease of sugarcane and Bermudagras stunting disease. International Journal of Systematic Bacteriology 34: 107117.

De León, L., F. Silverio, M.M. López \& A. Rodriguez. 2011. Clavibacter michiganensis subsp. michiganensis, a seedborne tomato pathogen: healthy seeds are still the goal. Plant Disease 95: 1328-1338.

De Pino, M. 2020. Guía didáctica: Cultivo y manejo del cultivo de tomate fresco. Curso de Horticultura y Floricultura, FCAyF, UNLP. $\quad$ Disponible https://aulavirtual.agro.unlp.edu.ar/pluginfile.php/47324/mod_folder/content/0/5.\%20Guia\%20de\%20To mate\%202020.pdf?forcedownload=1. Ultimo acceso: Octubre de 2021.

Di Rienzo, J.A., F. Casanoves, M.G. Balzarini, L. Gonzalez, M. Tablada \& C.W. Robledo. 2015. InfoStat versión. Grupo InfoStat, FCA, Universidad Nacional de Córdoba, Argentina. URL http://www.infostat.com.ar.

EPPO. 2013. Organisation Européenne et Mediterranéenne pour la Production des Plantes European and Mediterranean Plant Protection Organization. Clavibacter michiganensis subsp. michiganensis. Diagnostics PM 7/42. Bulletin OEPP/EPPO 43:46-67.

EPPO/ CABI. 1998. Map 253. In: Distribution Maps of Quarantine Pests for Europe. Smith IM, McNamara DG, Scott PR \& KM Harris Eds, CAB International, Wallingford (GB) 98 pp.

FAOSTAT. 2016. Food Agricultural Organization of the United Nations. http://www.fao.org/faostat/es/\#data/QC Último acceso: octubre de 2020.

Galeano, M., F. Mendez, \& A. Urbaneja. 2002. Efecto de Trichoderma harzianum Rifai (cepa T-22) sobre cultivos hortícolas. Koppert Biological Systems 286: 1-11.

Garat, J. 2002. Tomate platense en La Plata, Argentina. Biodiversidad 34: 19-21. 
García, M. 2011. El Cinturón hortícola platense: Ahogándonos en un mar de plásticos. Un ensayo acerca de la tecnología, el ambiente y la política. THEOMAI №23. Primer semestre 2011.

Gleason, M. L., R.D. Gitaitis \& M. Ricker. 1993. Recent progress in understanding and controlling bacterial canker of tomato in Eastern North America. Plant Disease 77: 1069-1076.

González, Y.P., Sifontes, J.L.A. \& M. Hurtado. 2013. Efecto bioestimulante de dos formulados líquidos de Trichoderma harzianum Rifai A-34 en la producción protegida de tomate el cultivo de tomate protegido. Centro Agrícola 40: 53-56.

Grondona, I., Hermosa R., Tejada M., Gomis M.D., Mateos P.F., Bridge P.D., Monte E. \& I. GarciaAcha. 1997. Physiological and biochemical characterisation of Trichoderma harzianum, as biological control agent against soilborne fungal plant pathogens. Applied and Environmental 63: 3189-3198.

Guerrero, R., C. Mónaco, M. Stocco, J. Rolleri \& N. Guerrero. 2017a. Selección de aislamientos de Trichodermaspp. para el control del cáncer bacteriano (Clavibacter michiganensis subsp. michiganensis) del tomate (Lycopersicum esculentum Mill.) Revista Amazónica Ciencia y Tecnología 6: 9-20.

Guerrero, R., Mónaco C., Stocco M., Consolo V., Rolleri J. \& N. Guerrero. 2017b. Aplicación de Trichoderma harzianum para el control del cáncer bacteriano (Clavibacter michiganensis subsp. michiganensis) del tomate (Lycopersicum esculentum Mill.) Revista Amazónica Ciencia y Tecnología 6: 230-243.

Harman, G., R. Howell, A. Viterbo, I. Chet \& M. Lorito. 2004. Trichoderma species. Opportunistic, avirulent plant symbionts" Nature Reviews. Microbiology 2: 43-56.

Hermosa, R., A. Viterbo, I. Chet \& E. Monte. 2012. Plant-beneficial effects of Trichoderma and of its genes. Microbiology 158: 17-25.

Hoitink, H., L. Madden \& A. Dorrance. 2006. Systemic resistance induced by Trichoderma spp.: interactions between the host, the pathogen, the biocontrol agent, and soil organic matter quality. Phytopathology 96: 186-189.

INTA \& CMCBA. 2009. Instituto Nacional de Tecnología Agropecuaria y Corporación del Mercado Central de Buenos Aires. 2009. Boletín electrónico de tomate $N^{\circ} 18$. Disponible en http://www.mercadocentral.gob.ar/boletin/pdf/Tomate18.pdf Último acceso: octubre de 2020.

Jiménez, C., N.S. de Albarracin, G. Altuna \& M. Alcano. 2011. Efecto de Trichoderma harzianum (Rifai) sobre el crecimiento de plantas de tomate (Lycopersicon esculentum L.). Revista de la Facultad de Agronomía LUZ 28: 1-10.

Kariuki, C.K., E.W. Mutitu \& W. M. Muiru. 2020. Effect of Bacillus and Trichoderma species in the management of the bacterial wilt of tomato (Lycopersicum esculentum) in the field. Egyptian Journal of Biological Pest Control 30: 4-8.

Kawaguchi A., K. Tanina \& K. Inoue. 2010. Molecular typing and spread of Clavibacter michiganensis subsp. michiganensis in Greenhouses in Japan. Plant Pathology 59:76-83.

Lo, C. \& C. Lin. 2002. Screening Strains of Trichoderma spp. for plant growth enhancement in Taiwan. Plant Pathology 11: 215-220.

Lorito, M., S. Woo, G. Harman \& E. Monte. 2010. Translational research on Trichoderma from omics to the field. Annual Review of Phytopahology 48: 395-417.

MDA. 2018. Ministerio Desarrollo Agrario de la provincia de Buenos Aires, Dirección de Economía, estadísticas y mercados. Area Teledetección y SIG. Cuantificación de superficie cubierta por invernáculos mediante Sensores Remotos satelitales. Informe preliminar. Disponible en file:///C:/Users/viver/Downloads/RESUMEN\%20GENERAL\%20INVERNACULOS\%20(1).pdf.

Montesinos, E. \& E. Bardají. 2008. Synthetic Antimicrobial Peptides as Agricultural Pesticides for PlantDisease Control. Chemistry \& Biodiversity: 5: 1225-1237.

Moo-Koh, F.A., J. Cristóbal-Alejo, A. Reyes-Ramírez, J.M. Tun Suárez \& M. Gamboa-Angulo. 2017. Identificación molecular de aislados de Trichodermaspp. y su actividad promotora en Solanum lycopersicum L. Investigación y Ciencia 25: 5-11.

Nawrocka, J. \& U. Malolepsza. 2013. Diversity in plant systemic resistance inducedby Trichoderma. Biological Control 67: 149-156.

Nguyen, M.T. \& S.L. Ranamukhaarachchi. 2010. Soil borne-antagonists for biological control of bacterial wilt disease caused by Ralstonia solanacearum in tomato and pepper. Journal of Plant Pathology 92: 395405. 
Noshad, A., M. Iqbal, L. Folkers, C. Hetherington, A. Aamir Khan, M. Numan \& S. Ullah. 2019. Antibacterial Effect of Silver Nanoparticles (AgNPs) Synthesized from Trichoderma harzianum against Clavibacter michiganensis. Journal of Nano Research 58: 10-19.

Otero, J., J.J. Garat, J. Vera Bahima, A. Ahumada, M.P. May \& A. Nico. 2014. Multiplicación, Estudio y Difusión de variedades hortícolas locales en el Cinturón Verde Platense. XVII Jornadas Nacionales de Extensión Rural y IX del Mercosur. El encuentro en la diversidad. Facultad de Ciencias Agrarias-UNR, Santa Fe. 19 al 21 de noviembre. Disponible en:https://www.researchgate.net/profile/JeremiasOtero/publication/317617962_MULTIPLICACION_ESTUDIO_Y_DIFUSION_DE_VARIEDADES_HORTI COLAS_LOCALES_EN_EL_CINTURON_VERDE_PLATENSE/links/5943d1ceaca2722db49d0633/MUL TIPLICACCION-ESTŪDIO-Y-DIFUSION-DE-VARIEDADES-HORTICOLAS-LOCALES-EN-EL-

CINTURON-VERDE-PLATENSE.pdf. Ultimo acceso: 30 de septiembre 2020.

Pastrik, K.H. \& F.A. Rainey. 1999. Identification and differentiation of Clavibacter michiganensis subspecies by polymerase chain reaction based techniques. Journal of Phytopathology 147: 687-693.

Peralta, I. E., S. Knapp \& D. Spooner. 2005. New species of wild tomatoes (Solanum section Lycopersicon: Solanaceae) from Northern Peru. Systematic Botany 30: 424-434.

Recinos-Cabrera, H.V. 2015. Evaluación de frecuencia de aplicación de Trichoderma harzianum sobre el desarrollo radicular de tomate. Salamá, baja Vera Paz. Tesis de Grado. Universidad Rafael Londívar. Facultad de Ciencias Ambientales y Agrícolas, Guatemala, 55 pp.

Rolleri, J. 2015. Cancro bacteriano del tomate: diagnóstico y prevención de su dispersión en el cultivo. M. Sc. Tesis. Facultad de Ciencias Agrarias y Forestales, Universidad Nacional de La Plata, La Plata, Argentina, $77 \mathrm{pp}$.

Samuels, G.J. 2006. Trichoderma: Systematics, the sexual state, and ecology. Phytopathology 96: 195206.

Schaad, N. W., J.B. Jones \& W. Chun. 2001. Laboratory guide for identification of plant pathogenic bacteria. American Phytopathological Society Press, St. Paul, 373 pp.

Shirakawa, T., T. Sasaki \& K. Ozaki. 1991. Ecology and control tomato bacterial canker and detection methods of this pathogens. Japan Agricultural Research Quarterly 25: 27-32.

Smith, E. F. 1910. A new tomato disease of economic importance. (Abstr.). Science (N.S.) 31: 794-796.

Stocco, M., A. Mansilla, C. Mónaco, C. Segarra, G. Lampugnani, C. Abramoff, M. Marchetti, N. Kripelz, C. Cordo \& V. Consolo. 2015. Native isolates of Trichoderma harzianum inducting resistance to Mycosphaerella graminicola, on wheat plants. Boletín de la Sociedad Argentina de Botánica 50: 291-301.

Stocco, M., C. Mónaco, C. Abramoff, G. Lampugnani, G. Salerno, N. Kripelz, C. Cordo \& V. F. Consolo. 2016. Selection and characterization of Argentine isolates of Trichoderma harzianum for effective biocontrol of Septoria leaf blotch of wheat. World Journal of Microbiology and Biotechnology 32: 2-10.

Stocco, M., G. Lampugnani, S. Zuluaga, C. Abramoff, C. Cordo \& C. Mónaco. 2019. Fungicida biológico a base de una cepa del hongo Trichoderma harzianum: su supervivencia en el suelo. Revista de la Facultad de Agronomía 118: 1-5.

Tancos, M. A., L. Chalupowicz, I. Barash, S. Manulis-Sasson \& C. D. Smart. 2013. Tomato fruit and seed colonization by Clavibacter michiganensis subsp. michiganensis through external and internal routes. Applied and Environmental Microbiology 79: 6948-6957.

Tucci, M., M. Ruocco, L. De Masi, M. De Palma \& M. Lorito. 2011. The beneficial effect of Trichoderma spp. on tomato is modulated by the plant genotype. Molecular Plant Pathology 12: 341354.

Utkhede, R. \& C. Koch. 2004. Biological treatments to control bacterial canker of greenhouse tomatoes. BioControl 49: 305-313.

Valdes Morales, M. 2016. Bacterias antagonistas para el control biológico de Ralstonia solanacearum (e. f. Smith) en tomate (Solanum lycopersici L.). Tesis para optar al título de Maestría en Ciencias. Coordinación Culiacán del CIAD en Ciencia y Tecnología para productos agrícolas de zonas tropicales y subtropicales. Ciad Repositorio, México, 83 pp.

Vinale, F. K., E.L. Sivasithamparam, R. Ghisalberti, S.L. Marra Woo \& M. Lorito. 2008. Trichoderma plant pathogen interactions. Soil Biology and Biochemistry 40: 1-10.

Volksch, B. \& R. May. 2001. Biological control of Pseudomonas syringae pv. glycinea by epiphytic bacteria under field conditions. Microbial Ecology 41: 132-139. 
Wilson, M., H.L. Campbell, P. Ji, J.B. Jones \& D. Cuppels. 2002. Biological control of bacterial speck of tomato under field conditions at several locations in North America. Phytopathology 92: 1284-1292.

Yedidia, I., A.K. Srivastva, Y. Kapulnik \& I. Chet. 2001. Effects of Trichoderma harzianum on microelement concentrations and increased growth of cucumber plants. Plant and Soil 235: 235-242.

Zahir, I., M. Babouchi, H. Boulanour \& M. El Louyti. 2018. Effet des microorganismes isolés á partir des biotipes Marocains sur les phytopatogénes. Revue bibliographique: Revue Agrobiologique 8: 971-983. 